\title{
Sarcopenia screening strategies in older people: a cost effectiveness analysis in Iran
}

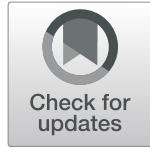

\author{
Ali Darvishi ${ }^{1,2}$, Mohsen Rezaei Hemami ${ }^{3}$, Gita Shafiee ${ }^{1 *}$, Rajabali Daroudii, ${ }^{4,2}$, Mahsa Mohseni ${ }^{2}$, \\ Farkhondeh Hosseini Shekarabi ${ }^{5}$ and Ramin Heshmat ${ }^{1}$
}

\begin{abstract}
Background and objectives: Sarcopenia is an important age-related disease which can lead to an increased risk of mortality, falls, fractures, and poor quality of life. So, timely detection can be effective in reducing the burden of disease. The aim of this study was to identify the most cost-effective strategy for sarcopenia screening in Iran.

Materials and methods: We constructed a Markov transition model over a life-time horizon based on natural history. Compared strategies included Sarcopenia scoring assessment models (SarSA-Mod), European working group on sarcopenia in older people (EWGSOP), Mini sarcopenia risk assessment (MSRA) and SARC-F. Parameters values were extracted from primary data and the literature, and the costs and Quality-adjusted life years (QALYs) were calculated for each strategy. Sensitivity analysis of uncertain parameters was also performed to determine the robustness of the model. Analysis was performed using 2020 version of TreeAge Pro software.
\end{abstract}

Results: All four screening strategies increased life time QALYs. After removing dominated strategy, the incremental cost per QALY gained for sarcopenia screening varied from $\$ 1875.67$ for EWGSOP to $\$ 1898.33$ for MSRA. Our basecase analysis showed that the most cost-effective strategy was EWGSOP and 2nd best was SarSA-Mod with \$43, 414.3 and $\$ 42,663.3$ net monetary benefits given one GDP per capita (\$5520.311) as willingness to pay, respectively. Sensitivity analysis of model parameters also showed robustness of results.

Conclusions: The results of the study, as the first economic evaluation of sarcopenia screening, showed that the EWGSOP strategy is more cost-effective than other strategies.

Keywords: Sarcopenia, Screening, Cost effectiveness analysis (CEA), QALY

\section{Introduction}

One of the important topics in geriatric medicine is sarcopenia or loss of age-related skeletal muscles that has been considered more in recent years [1,2]. Sarcopenia is defined as syndrome with the loss of progressive skeletal muscle mass and function that is significantly accompanied with increasing mortality, reducing quality of life, and increasing costs of the elderly care [3, 4]. During the years of 50 to 80 of life, sarcopenia reduces muscle

\footnotetext{
* Correspondence: gshafiee.endocrine@gmail.com

${ }^{1}$ Chronic Diseases Research Center, Endocrinology and Metabolism Population Sciences Institute, Tehran University of Medical Sciences (TUMS), NO 10, Jalale-Al-Ahmad Ave, Chamran Highway, Tehran 1411713137, Iran Full list of author information is available at the end of the article
}

mass by about 30\% [4] and is accompanied with myasthenia, increasing fatigue and inappropriate function in elderly which may cause to reduce individual's ability in moving quickly and increase the risk of falling $[5,6]$.

Sarcopenia can harms on society health and welfare and on the other hand it leads to increase the health costs, the risk of physical disability, reducing the quality of life, increase the need for care services, and ultimately increase the risk of mortality in elderly age groups. Therefore, in recent decades it has attracted the attention of researchers around the world [4, 6-8]. Impotence and dependency in personal affairs, falling and fracture are the most important complications that have led to

(c) The Author(s). 2021 Open Access This article is licensed under a Creative Commons Attribution 4.0 International License, which permits use, sharing, adaptation, distribution and reproduction in any medium or format, as long as you give appropriate credit to the original author(s) and the source, provide a link to the Creative Commons licence, and indicate if changes were made. The images or other third party material in this article are included in the article's Creative Commons licence, unless indicated otherwise in a credit line to the material. If material is not included in the article's Creative Commons licence and your intended use is not permitted by statutory regulation or exceeds the permitted use, you will need to obtain permission directly from the copyright holder. To view a copy of this licence, visit http://creativecommons.org/licenses/by/4.0/ The Creative Commons Public Domain Dedication waiver (http://creativecommons.org/publicdomain/zero/1.0/) applies to the data made available in this article, unless otherwise stated in a credit line to the data. 
consider osteoporosis and sarcopenia as a movement disorder syndrome [9]. In addition, the results of studies have shown that sarcopenia is associated with other diseases such as metabolic syndrome, respiratory diseases as well as cardiovascular diseases (CVDs) [10-12]. Considering all the different aspects of the disease, sarcopenia can have a huge economic burden on the health care system and society welfare.

It is estimated that sarcopenia occurs in 5 to $45 \%$ of the elderly population [13-16]. Although sarcopenia occurs mainly in the elderly, there are also cases in younger people. A study conducted by Cherin revealed that $9 \%$ of individuals between the ages of 45 to 54 years and $13.5 \%$ of people aged 55 to 64 are sarcopenic [17]. Other studies have shown that the rate of sarcopenia is very common in elderly hospitalized patients and is about 10 to $37.3 \%$, of which about one-fifth of these patients are under 65 years old [18-21]. The results of sarcopenia frequency assessment in Iran based on the definition of the EWGSOP for 300 people of over the age of 55 years showed that $30 \%$ of men and $18 \%$ of women are sarcopenic [22-24]. The prevalence of sarcopenia is related to the type of muscle mass measuring instrument [2]. Available instruments include bioimpedance analysis (BIA) and Dual-energy X-ray absorptiometry (DXA) which have different measurement accuracy and cost. A review of studies shows that in general, the prevalence of sarcopenia based on BIA instrument is estimated higher than DXA and in other words the prevalence is overestimated by using the BIA instrument [2].

Prevalence of the sarcopenia is high in Iran, as well as complications and consequences of this disease is considerable, so screening at-risk individuals and also performing effective interventions at the beginning of the disease progression could have a significant impact on reducing the negative consequences of this disease. Therefore, in the early stages of the disease, that the functional disorder has not yet occurred, there may be a good opportunity to perform interventions such as supplements, nutritional diet and exercise to reduce the progression of the disease [25-27].

Screening strategies and instruments are used in the early detection of at risk people for sarcopenia in the clinical and research section. Overall, existing screening strategies are screening questionnaire, diagnostic algorithms, or predictive equations [2]. There is a wide range of sarcopenia measurement components including body muscle mass and muscle strength and performance whereas the most important symptom of sarcopenia is reducing skeletal muscle mass [2]. Different methods are vary in accuracy, cost, and duration of measurement. In many countries, it is not possible to perform these tests especially examining the muscle mass measurement by DXA. Therefore, researchers seek easier ways for assessing and screening of sarcopenia [2]. The first screening strategy was presented by EWGSOP in 2010, which is a two-step algorithm [28]. The Asian working group for sarcopenia (AWGS) also provided an algorithm in order to screen sarcopenia in the elderly people in 2014 [2]. A questionnaire called SARC-F was also designed by Malmstrom and Morley, which consists of five sections. This questionnaire can detect older people who need to additional assessment for sarcopenia (high-risk individuals) [29]. Ishii and colleagues (2014) innovated another method for detection of the elderly people at risk of sarcopenia that can estimate possibility of sarcopenia [26]. Yu and his colleagues in Australia, using a predictive equation of the anthropometry components with muscle strength, can detect people who are at risk of sarcopenia [30]. Mini sarcopenia risk assessment (MSRA) questionnaire is also designed by Rossi et al. which can detect at risk people [31]. A model called sarcopenia scoring assessment model (SarSA-Mod) has been recently designed in Iran that can help to detect people at risk of sarcopenia with high accuracy [32].

The existing information on economic burden of sarcopenia is limited and not many studies have been conducted on this subject. A study, that is conducted on a sample of people over than 60 years old in USA in 2004, has shown that the health care cost related to sarcopenia is about \$ 18.5 billion (\$ 10.8 billion for men and \$ 7.7 billion for women) [33]. Considering population aging as well as the health and economic side effects of the disease progression and limitation of health care financial sources, so the expanding the screening for sarcopenic people and early detection of at-risk individuals and administering timely interventions are very important. On the other hand, considering that there are different screening methods with various costs and effectiveness, the present study aims to determine the cost and effectiveness of sarcopenia screening strategies in Iran and tries to provide the evidence and information needed for policy and decision making for applying best strategy in this regard.

\section{Materials and methods}

The present study is a full economic evaluation which was made a comparison of four sarcopenia screening strategies as well as no screening. Study was conducted in two phases. In order to review available evidences in this subject, in first phase a systematic review study of economic evaluation studies for sarcopenia screening was conducted to examine how to evaluate parameters and challenges ahead and use of its results in designing economic evaluation model. However, economic evaluation study related to sarcopenia was not found based on our inclusion criteria (systematic review results of the first phase of the study could be seen in the form of the 
Prisma flow diagram in the supplementary information (Fig. S1). Therefore, in phase two of the study, for the first time, we developed a cost-effectiveness analysis model to compare sarcopenia screening strategies.

\section{Modeling}

Designing cost-effectiveness analysis model was conducted based on the natural history of disease, how and the protocol of performing the four screening methods, the clinical outcomes, the probabilities of the occurrence of the consequences and the occurrence of the costs. In order to design the model, specialized panels were formed with the participation of the study team of clinical experts and the economic team. After the trial and error of different models, the final model was extracted with consensus and considering the most important clinical and economic outcomes based on the diagnosis and treatment of disease natural history.

According to the review of the clinical evidence of the disease and also the results of the expert opinions, four variables were finally considered as the intermediate outcome. Increasing the risk of falling and consequently the risk of fracture, increasing the risk of CVDs due to sarcopenia, increasing the risk of death and reducing the quality of life were considered as the main outcome of the model. Comorbidities have also been omitted due to the simplification of the model and the ability to evaluate.

Structure of the model was designed in two parts: the decision tree and markov transition model. Decision tree is developed based on the evidence and process of screening tests. In this model, individuals enter to decision tree and the process of events is considered the same in each of the strategies. After entering the model, individuals are divided into two groups according to the prevalence of sarcopenia: sarcopenic and healthy. In each of the two groups, the test results could be positive or negative (false and true positives or false and true negatives) (Fig. 1). Then all the groups enter the Markov model depending on whether they are sarcopenic or healthy.

\section{Markov model}

The schematic and simple structure of the Markov model used in this study can be seen in Fig. 2. We considered 1000 study populations in the hypothetical Markov cohort.

The model consists of three health states: sarcopenia without cardiovascular disease, sarcopenia with cardiovascular disease and death, and individuals are in transition during the simulation and study period according to the probability of transition between these conditions so that at the end of each cycle individuals remain in the same health state or are transferred to other health state, and eventually in the final cycle all individuals transferred to death health state.

\section{Model assumptions}

Our model has the following rules and assumptions:

Each strategy, regardless of its stages, acts as a single test, which ultimately has a positive or negative response, which is classified as true and false positives and true and false negatives.

The progression of the disease in Markov's structure is forward-looking for more simplicity and is considered irreversible.

In this model, the effectiveness of timely diagnosis and treatment is considered in the probability of transitions between states as well as the risk of occurrence of outcomes such as the risk of falls and fractures.

In false positive cases, patients are examined clinically and undergo a therapeutic intervention cycle, and this is the only additional cost that is imposed on this group of patients.

Markov's time horizon, with regard to the life expectancy of Iranian men and women aged 60 years, was considered to be 25 years. The length of Markov cycles is also considered to be 1 year due to the nature of the disease state of changes. Since the individuals could survive less than 1 year, we apply the half-cycle correction to adjust for the potential overestimation of the costs and utilities.

It should be noted that sarcopenic patients are usually divided into three groups: Pre-sarcopenia, sarcopenia and severe sarcopenia [34], but in the current Markov structure, the patients are considered all in one group and the health state of the accumulated sarcopenia and the division of patients into three groups has been discarded due to changes in definitions of pre-sarcopenia in recent years and the disagreement over them and the lack of access to the separate evidence $[35,36]$.

In the present model, fall and fracture are considered an event in life cycles. Due to this, after fall and fracture people have a lower quality of life and also incur new treatment costs.

Moreover, in this model, regarding the available limitations, it is assumed that screening and diagnosis are performed only once, and screening is not performed during Markov cycles, and costs have not been considered in this regard.

\section{Screening strategies and descriptions}

In present cost-effectiveness analysis, we compared four sarcopenia screening strategies with the no screening strategy. The selection of screening strategies was based on Iran health care setting and which are used for sarcopenia screening in Iran and a strategy has been added to 


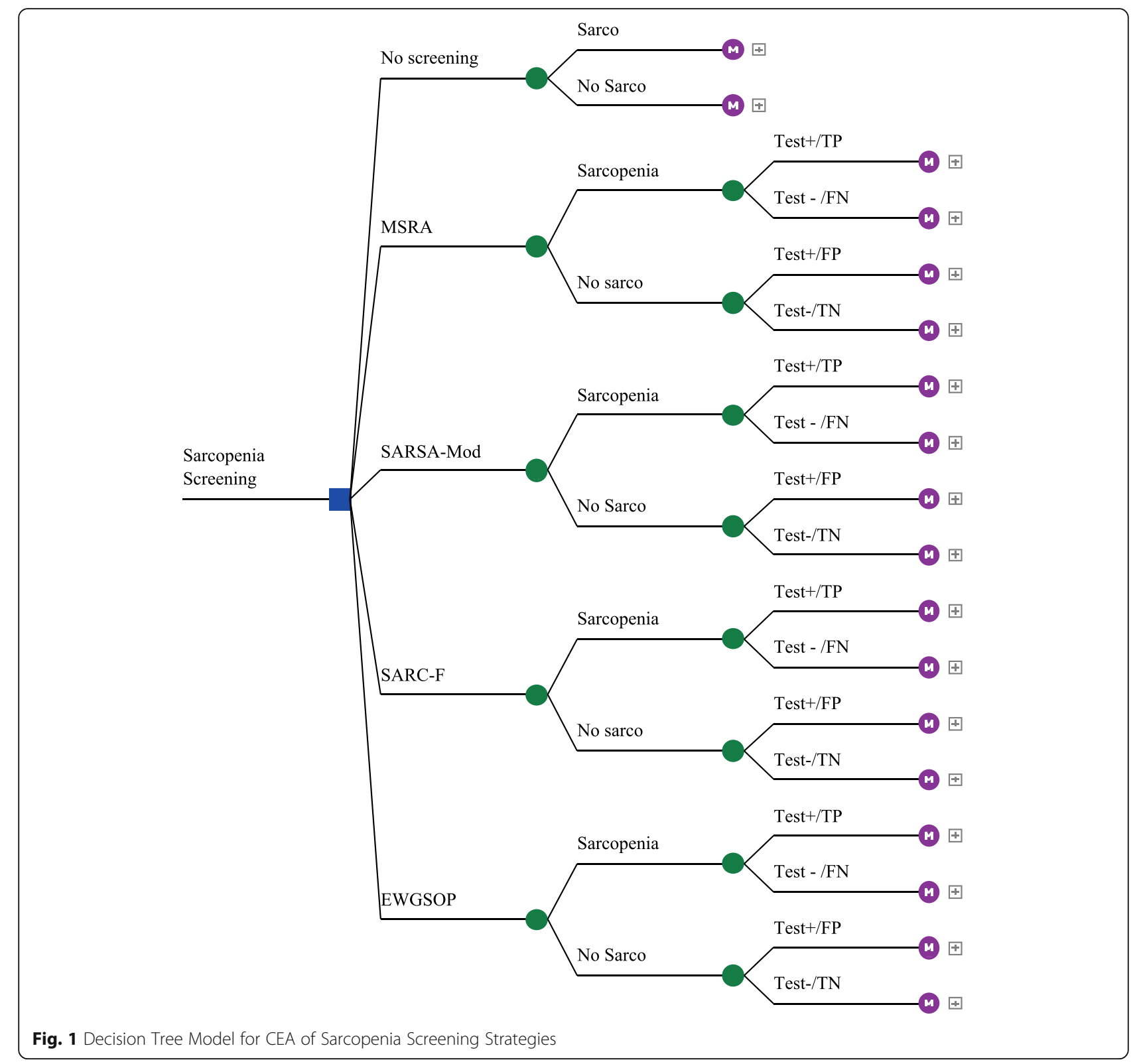

this comparison as a new method for sarcopenia screening.

1. The European Working Group on Sarcopenia in Older People (EWGSOP) algorithm: This method is done in two parts. In the first part, the muscle performance test and handgrip strength test are performed, and in the second part, according to the defined cut of points for the at-risk individuals in the first part, the muscle mass measurement is preferably performed using DXA [28].

2. SARC-F method: This method is based on a questionnaire designed by Malmstrom and
Morley, which includes 5 parts. This questionnaire can detect elderly people who need supplementary assessment for sarcopenia (highrisk individuals) [29].

3. Mini Sarcopenia Risk Assessment (MSRA) method: This method is also based on the MSRA questionnaire designed by Rossi and his colleagues that can identify high-risk individuals. The questionnaire includes seven items including age, level of physical activity, hospitalization, weight loss, and three dietary questions [31].

4. Sarcopenia Scoring Assessment Models (SarSAMod) method: A model called SarSA-Mod has been designed which can identify sarcopenia at risk 


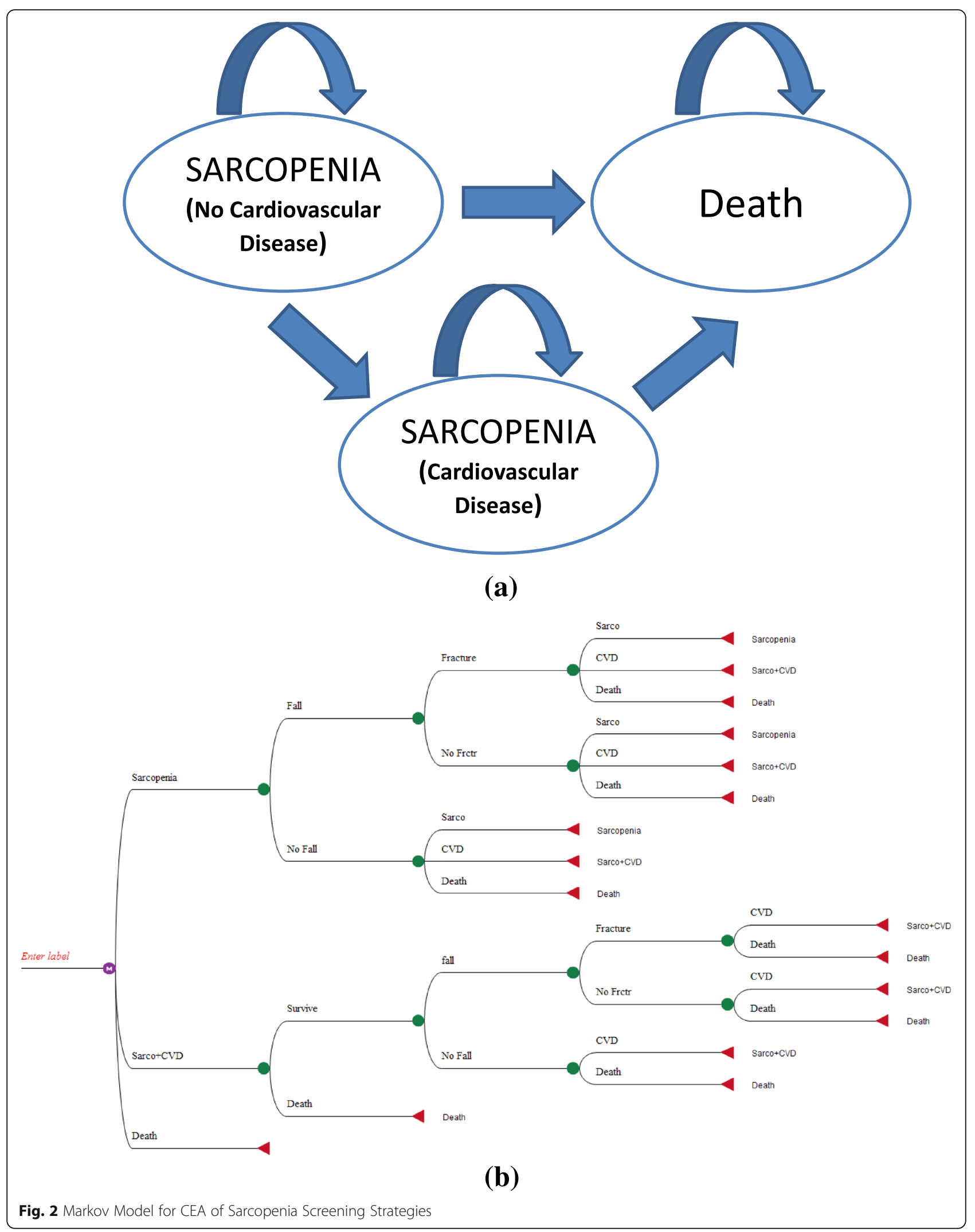


individuals. In this model, it evaluates the variables of age, weight, and calf circumference for men and women. In brief, to develop a statistical screening model to identify patients with sarcopenia, the study sample was randomly divided into two parts; $67 \%$ of the cases, called development set, which were allocated to the development of the model, and $33 \%$ of the dataset, named validation set were allocated to validation of the model. Logistic regression analysis with stepwise approaches was applied in the development of the model. $ß$ coefficient of each variable was used to calculate its index weight. The ability of the model to separate those with sarcopenia from those without sarcopenia was evaluated using receiver operating characteristic (ROC) curves and the area under the ROC curve. A suitable cut-off point was selected with regards to sensitivity and specificity. Then, sensitivity, specificity, positive and negative predictive values (PPV \& NPV) and the accuracy of the scores were evaluated using the validation set, which was left aside so far and not engaged in the model development process. In order to select and validate the final criteria for our scoring model, the model was applied to the validation set, using ROC analyses [unpublished data].

5. No screening.

\section{Extracting parameter values and how to analyze model} Parameters and variables related to screening strategies such as sensitivity, specificity, sarcopenia prevalence, probability of transitions, risks of fall and fracture and other parameters were extracted from the literatures. In this regard, a separate each discrete search parameter was performed based on keywords and specific search strategy in scientific databases, and studies that had appropriate evidence were classified, and finally the best available evidence was extracted. In cases where there was no evidence for the desired parameter, the values of the parameters were estimated using the experts' opinion (panel including clinical specialists and economic team) (Table 1).

Detailed information about sensitivity and specificity values of screening strategies are presented in Table S1.

\section{Utility}

The primary outcome of the study is the number of identified sarcopenia cases. Because the present study is a full economic evaluation study, the final outcome in this evaluation is considered the Quality Adjusted Life Years Index (QALYs) and each screening strategy are finally evaluated based on cost per QALY unit. Evidence of quality of life and utility in any health state has been extracted from international studies (Table 1).

\section{Cost}

The study was conducted from the health system perspective, and the time horizon, as mentioned, was considered 25 years, and the costs were estimated accordingly.

The cost of each screening strategy, including the cost of screening and diagnostic tests, is calculated based on the cost unit used in each intervention. Gathered data from internal and external related evidence were used to do this. Additionally, the cost of treatment interventions for sarcopenia, the cost of treatment and care for fractures and the cost of treatment of cardiovascular diseases were calculated based on treatment protocols, the opinions of a specialized team and based on public tariffs of the Ministry of Health of Iran in 2019.

All Costs were converted from Iran Rial to the US Dollar at the official currency rate of 42,000 Rial per Dollar.

\section{Cost-effectiveness analysis}

Finally, in order to analyze and assess the cost effectiveness strategy, the incremental cost-effectiveness ratio (ICER) was used. The formula for this index is as follows [51]:

$$
I C E R=C_{1}-C_{2} / E_{1}-E_{2}
$$

In this regard, $\mathrm{C}$ represents the cost of strategies and $\mathrm{E}$ indicates the value of effectiveness of the strategies.

After calculating the ICER, the strategy with the lowest ICER is introduced as the most cost effective strategy.

We also used the threshold value equal to the Islamic Republic of Iran (IRI) one GDP per capita (\$5520.311) introduced by the WHO as the cost effectiveness threshold (Willingness to pay) to identify the most cost effectiveness strategy.

\section{Sensitivity analysis}

With regard to the uncertainty about some of the parameters used in the model, the deterministic sensitivity analysis (DSA) and the probabilistic sensitivity analysis (PSA) of the base case results were conducted. In the present study, first, a one-way deterministic Sensitivity Analysis was performed using tornado diagram for all uncertain parameters including sarcopenia prevalence, sensitivity and specificity of tests, test costs, treatment of fracture cost, interventions' cost was estimated. Since the deterministic sensitivity analysis cannot fully demonstrate all the uncertainties in the parameters used, the PSA was also used. Thus, considering the possible distribution of uncertain variables using Monte Carlo Simulation, cost effectiveness acceptability curve, incremental cost effectiveness scatter plot and other reports were extracted. Distributions used in PSA are shown in Table 1. 
Table 1 Main input parameters of CEA model

\begin{tabular}{|c|c|c|c|c|}
\hline Statistic variable & Base case & $\mathrm{SD} /(\mathrm{Cl})$ & Distribution & Source \\
\hline Sarcopenia prevalence in Iran & 0.245 & \pm 0.08 & Beta & [24] \\
\hline Annual discount rate & 0.05 & $(0.03-0.12)$ & Beta & \\
\hline Time Horizon (years) & 25 & & & \\
\hline \multicolumn{5}{|l|}{ Sensitivity of screening tests } \\
\hline EWGSOP & 1 & - & Beta & [32] and Table S1 \\
\hline SarSA-Mod & 0.806 & $(0.767-0.842)$ & Beta & [32] and Table S1 \\
\hline MSRA & 0.804 & \pm 0.08 & Beta & [31] \\
\hline SARC-F & 0.168 & $(0.135-0.206)$ & Beta & [32] and Table S1 \\
\hline \multicolumn{5}{|l|}{ Specificity of screening tests } \\
\hline EWGSOP & 0.84 & $(0.816-0.862)$ & Beta & [32] and Table S1 \\
\hline SarSA-Mod & 0.788 & $(0.75-0.803)$ & Beta & [32] and Table S1 \\
\hline MSRA & 0.604 & $(0.543-0.664)$ & Beta & [31] \\
\hline SARC-F & 0.866 & $(0.843-0.886)$ & Beta & [32] and Table S1 \\
\hline \multicolumn{5}{|l|}{ Probability of transition } \\
\hline $\begin{array}{l}\text { Probability of death in non-sarcopenic individuals } \\
\text { (Normal Pop } 60 \text { yrs) }\end{array}$ & 0.09 & & Beta & IRI Life Table \\
\hline Probability of death in sarcopenic individuals & 0.132 & \pm 0.013 & Beta & {$[37,38]$} \\
\hline $\begin{array}{l}\text { Probability of death in sarcopenic individuals with } \\
\text { cardiovascular disease }\end{array}$ & 0.198 & \pm 0.03 & Beta & {$[37,38]$ And Calibration } \\
\hline $\begin{array}{l}\text { Probability of death for sarcopenic individuals } \\
\text { with fracture }\end{array}$ & 0.171 & \pm 0.02 & Beta & {$[37,38]$ And Experts opinion } \\
\hline Probability of falling in sarcopenic individuals & 0.273 & \pm 0.03 & Beta & [39-41] \\
\hline Probability of fracture in sarcopenic individuals & 0.40 & \pm 0.05 & Beta & [37] \\
\hline $\begin{array}{l}\text { Probability of cardiovascular disease (CVDs) in } \\
\text { sarcopenic individuals }\end{array}$ & 0.27 & \pm 0.03 & Beta & [12] And Experts opinion \\
\hline \multicolumn{5}{|l|}{$\operatorname{costs}(\$)$} \\
\hline $\begin{array}{l}\text { Annual average cost of treatment interventions } \\
\text { (vitamin D, Supplements, exercise, diet) }\end{array}$ & 1119.048 & \pm 119.047 & Gamma & Survey and Calibration \\
\hline Cost of usual gait speed test & 0.714 & & Gamma & Survey and Calibration \\
\hline Cost of handgrip strength test & 1.309 & & Gamma & Survey and Calibration \\
\hline Cost of DXA & 42.857 & & Gamma & Survey and Calibration \\
\hline Cost of MSRA & 0.714 & & Gamma & Survey and Calibration \\
\hline Cost of SARC-F & 0.714 & & Gamma & Survey and Calibration \\
\hline Cost of SARC-F (high-risk individuals) & 44.880 & & Gamma & Survey and Calibration \\
\hline Cost of SarSA-mod & 1.785 & & Gamma & Survey and Calibration \\
\hline EWGSOP (stage 1) & 2.023 & & Gamma & Survey and Calibration \\
\hline EWGSOP (stage 2) & 42.857 & & Gamma & Survey and Calibration \\
\hline Cost of treatment and care of fractures & 3599.048 & \pm 695.074 & Gamma & Survey and Calibration \\
\hline $\begin{array}{l}\text { The initial average per capita cost of treatment } \\
\text { and care of cardiovascular patients }\end{array}$ & 4149.286 & & Gamma & Survey and Calibration \\
\hline $\begin{array}{l}\text { The incremental average per capita cost of treatment } \\
\text { and care of cardiovascular patients }\end{array}$ & 1904.762 & \pm 190.476 & Gamma & Survey and Calibration \\
\hline \multicolumn{5}{|l|}{ Utilities } \\
\hline Non-sarcopenic individuals (Normal Pop > 60 yrs) & 0.76 & & Beta & {$[42,43]$} \\
\hline Sarcopenic individuals & 0.68 & & Beta & [44] \\
\hline Sarcopenic individuals with fracture & 0.51 & & Beta & {$[40,44,45]$} \\
\hline Sarcopenic individuals with cardiovascular disease & 0.56 & & Beta & {$[44,46]$} \\
\hline Sarcopenic individuals with cardiovascular disease and fracture & 0.42 & & Beta & [44-47] \\
\hline \multicolumn{5}{|l|}{ Efficacy of interventions } \\
\hline Efficacy of sarcopenia interventions (vitamin D, Supplements, exercise, diet) & $40 \%$ & $\pm 15 \%$ & Beta & [48-50] \\
\hline
\end{tabular}


Table 2 Results of base case cost-effectiveness analysis

\begin{tabular}{lllllll}
\hline Strategy & Cost(\$) & ${\text { Incr } \operatorname{Cost}(\$)^{\mathbf{a}}}^{\mathbf{a}}$ & $\mathbf{E f f}^{\mathbf{b}}$ & Incr Eff $^{\mathbf{c}}$ & ICER(\$) $^{\text {Special }}$ \\
\hline No screening & 1455.07 & 0 & 7.40 & 0 & 0 & Base line \\
SARC-F & 1809.41 & 354.34 & 7.59 & 0.19 & 1897.84 \\
SARSA-Mod & 3140.83 & 1685.76 & 8.30 & 0.90 & 1881.96 \\
MSRA & 3151.27 & 1696.20 & 8.30 & 0.89 & 1898.33 \\
EWGSOP & 3518.75 & 2063.68 & 8.50 & 1.10 & 1875.67 \\
\hline
\end{tabular}

${ }^{a}$ Incremental costs, ${ }^{b}$ Effectiveness, ${ }^{c}$ Incremental Effectiveness

In cases where no evidence was found for the distribution of the parameter, $10-15 \%$ of the mean parameter value was considered as the standard deviation and appropriate distribution was selected according to the type of variable.

Modeling and analysis of base case results, as well as all stages of sensitivity analyses were performed using the 2020 version of Tree Age software.

\section{Results}

\section{Base case}

The results of the base case analysis can be seen in Table 2.

In Table 2, screening strategies are arranged from the lowest to the highest life time average costs, respectively, and the incremental cost, incremental effectiveness, and incremental cost-effectiveness ratio (ICER) were also calculated. No Screening was considered as the reference strategy for calculation of ICER. The ICER of the EWGSOP strategy was the lowest ICER value among the 5 strategies equivalent of $\$ 1875.67$ per QALY units.
Considering the cost-effectiveness threshold of $\$ 5520.311$ (equal to Iran GDP per capita in 2017), Net Monetary Benefits of EWGSOP, SarSA-Mod, MSRA, SARC-F and No Screening strategies were estimated $\$ 43$, $414.3, \$ 42,663.3, \$ 42,640.6, \$ 40,080.6$ and $\$ 39,404.3$ respectively, which shows that after EWGSOP, using SarSAMod strategy has the highest net monetary benefits.

Figure 3 shows the cost effectiveness plan of the study, which shows the placement of each of the strategies compared in this plot. Curve-based strategies are dominant and have lower cost and greater effectiveness (No screening, SARC-F, EWGSOP), and strategies that are outside of this curve are dominated strategies.

\section{Sensitivity analysis}

Results of sensitivity analysis were performed in two sections: DSA and PSA which described separately.

\section{Deterministic sensitivity analysis (DSA)}

In this section, the deterministic sensitive analysis was performed using one-way sensitivity analysis and

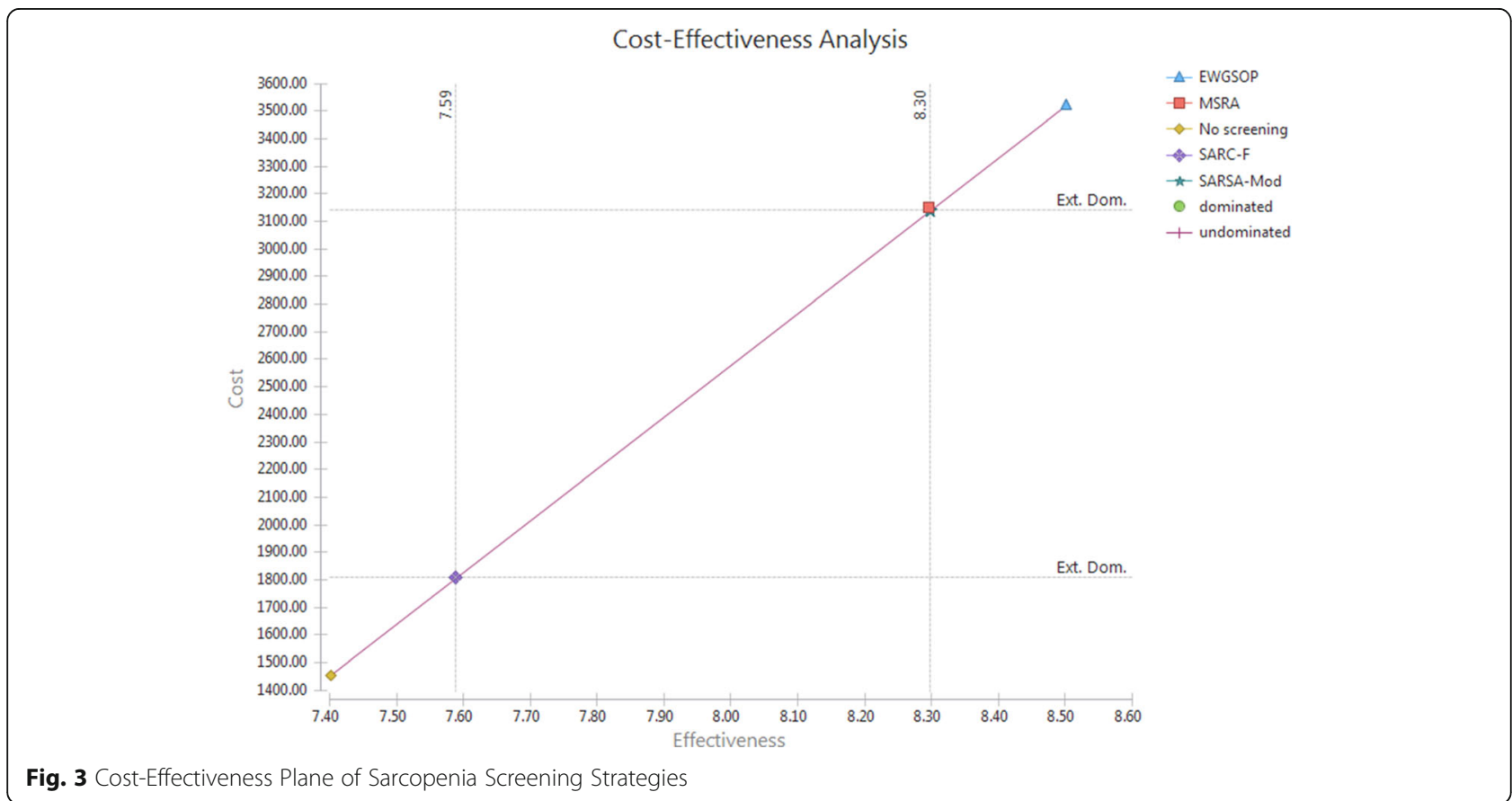




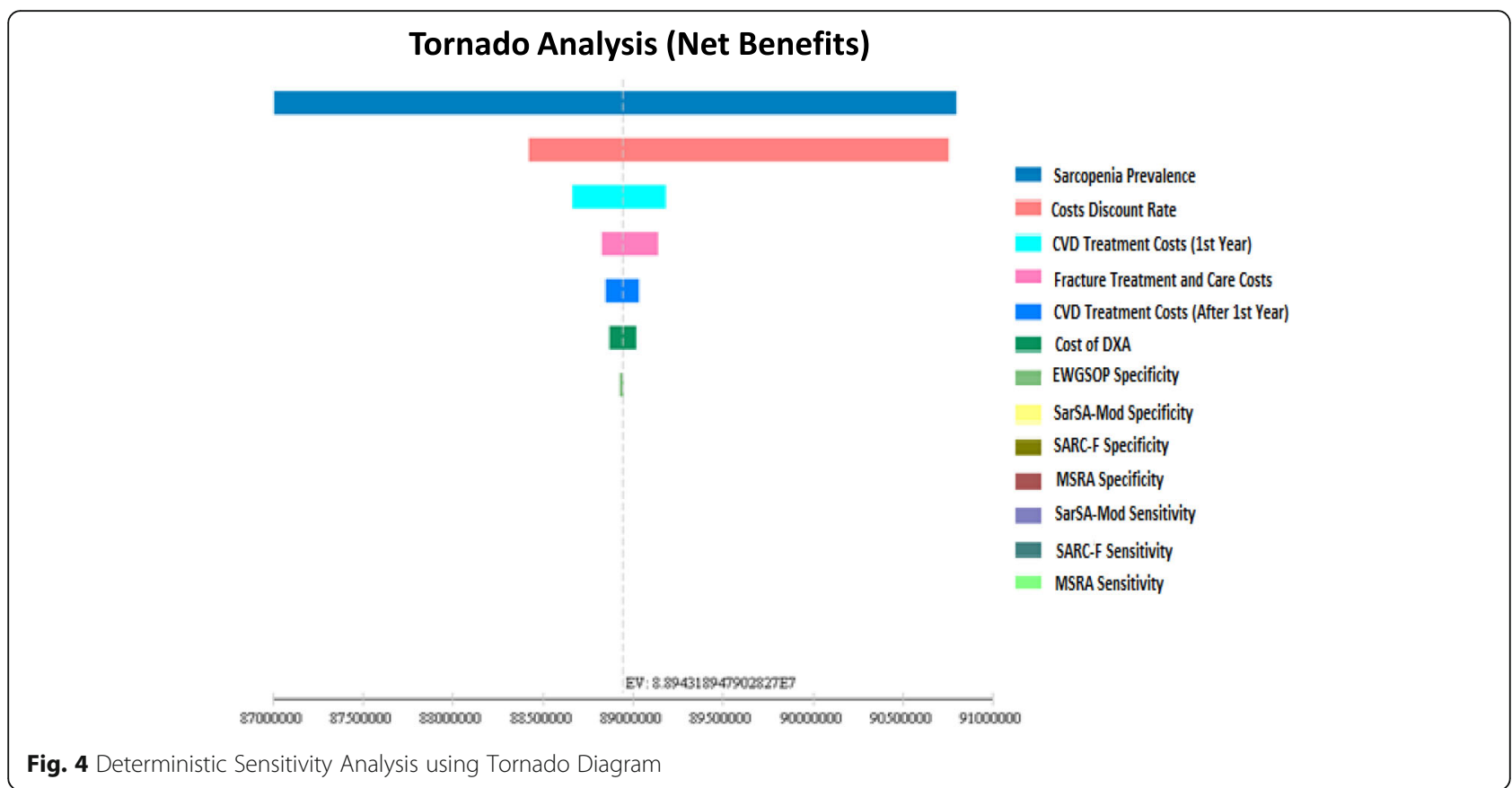

tornado diagram. Accordingly, the rate of the sensitivity of the model results to uncertain parameters changes was measured. Sensitivity analysis results can be seen using a tornado diagram in Fig. 4. As shown, the results of the study are most sensitive to changes in the parameters of the sarcopenia prevalence and discount rate of sarcopenia treatment, CVDs and fracture costs.

It should be noted that although the changes in these parameters have changed the results of the model, the overall results of cost-effectiveness is not sensitive to changes in any of the uncertain parameters, in other words, these parameters do not have a threshold.

In general, the results of deterministic sensitivity analysis showed that the results of the base case are robust.

\section{Probabilistic sensitivity analysis (PSA)}

PSA was performed using Monte Carlo Simulation regarding 1000 times of sampling replications.

Accordingly, the cost effectiveness acceptability curve (CEAC) was extracted (Fig. 5). As can be seen, increasing the threshold value increases the probability that the

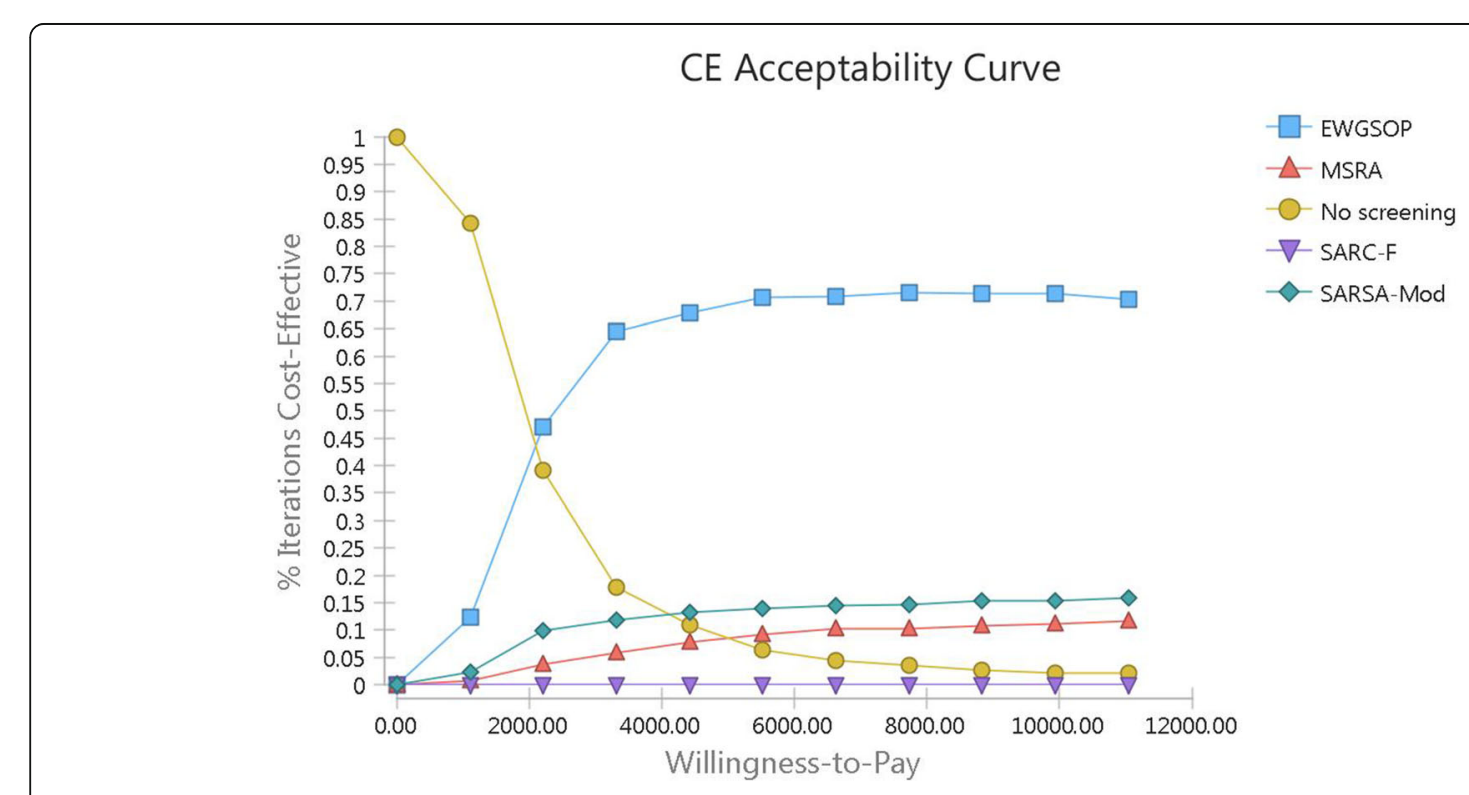

Fig. 5 Cost Effectiveness Acceptability Curve of Sarcopenia Screening Strategies 


\section{Monte Carlo Strategy Selection (WTP: 5520.311)}

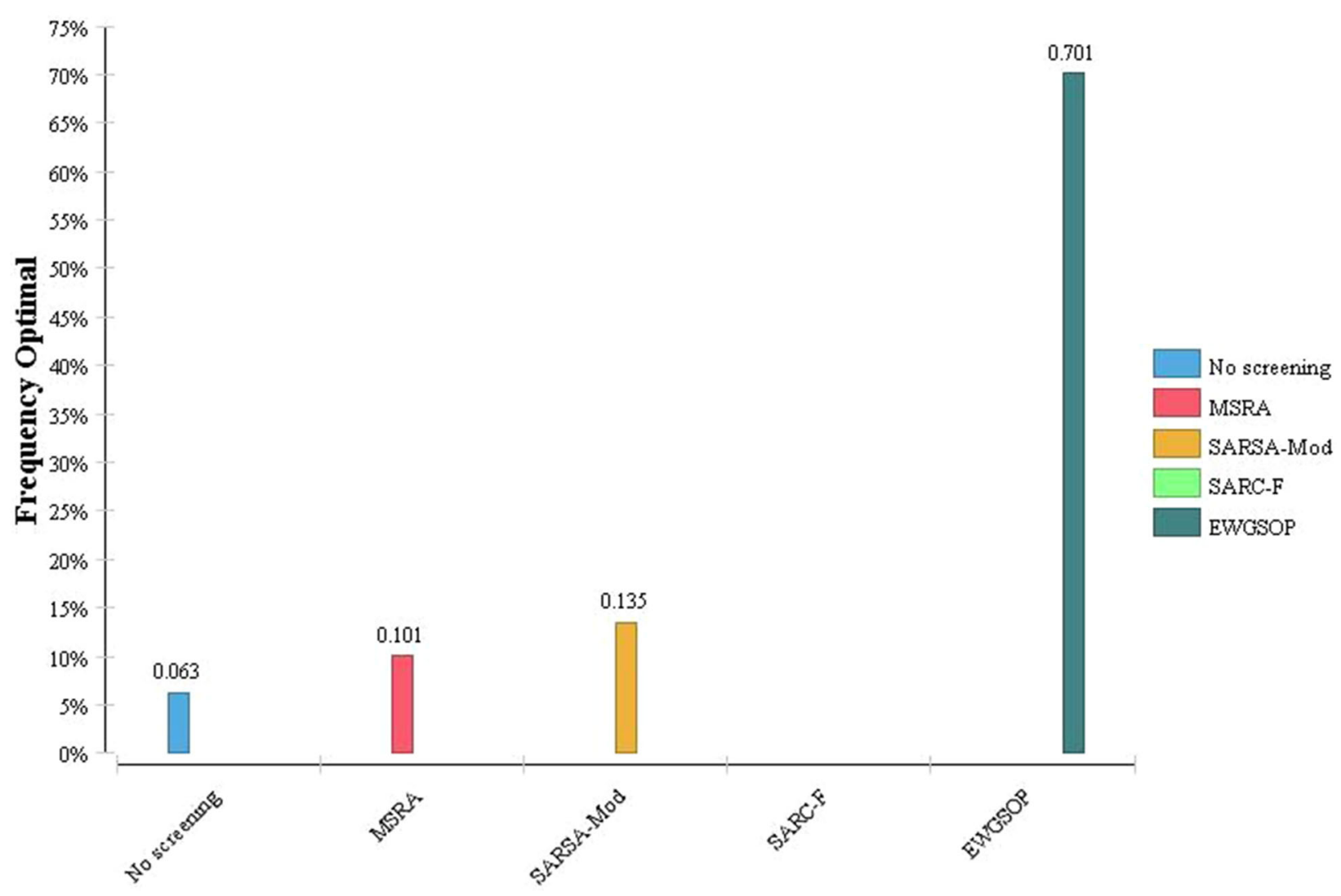

Fig. 6 Strategy Selection and Optimization Probability Diagram

EWGSOP strategy will be cost effective and it reduces the probability of cost-effectiveness of the non-screening strategy. In addition, the probability of cost-effectiveness of other strategies in all threshold values is about less than $20 \%$.

The above results can also be deduced from the strategy selection diagram (Fig. 6). As can be seen, considering the cost-effectiveness threshold of $\$ 5520.311$ and 1000 times of sampling replication in Monte Carlo simulation, the probability of cost-effectiveness of EWGSOP strategy is 70\% and the chances of other strategies including SarSA-Mod, no screening and MSRA are 13, 10, and 6\%, respectively.

All of the PSA results and Monte Carlo simulation showed that the results of the base case are highly probable robust.

\section{Discussion}

The present study is a full economic evaluation was conducted to compare different strategies of sarcopenia screening. The results of the base case analysis indicated that the EWGSOP strategy was the most cost-effective strategy from a health system perspective. DSA and PSA showed that the base case results were highly robust. It also seems that due to the possibility of identifying patients with high accuracy using this screening method and using timely treatment interventions, higher effectiveness can be achieved than other strategies.

The feasibility of EWGSOP method in all geographical areas of a society is very important. Given that one of the parts in the EWGSOP strategy is to use a DXA bone densitometry device to confirm the diagnosis of sarcopenia [28]. Due to the high budget impact of the DXA, in the short term, it is not possible to use this device in many geographical areas and non-DXA-based methods for sarcopenia screening should be used. The results of this study showed that among the compared strategies, the SarSA-Mod method is the best strategy after EWGSOP in terms of cost effectiveness. With regard to the relatively appropriate sensitivity of this method, the use of simple variables to identify patients, as well as the low budget impact of this strategy, it seems that this method can be used at some levels to identify early at risk sarcopenic individuals. In general, according to the results of 
the study, the best way to identify early sarcopenic individuals, in areas having DXA devices, is the EWGSOP method, and on the contrary, in areas do not have DXA and areas where the establishment of this device is not cost-effective using simpler screening tools to diagnosis and timely intervene for preventing disease progression is very important, and as the results showed, the SarSAMod strategy seems to be the best choice.

With regard to the growing trend of aging in the world, it is important to pay attention to diseases related to elderly ages that timely diagnosis of diseases can assist to reduce overall costs of the health system and also improve the individuals' quality of life. Sarcopenia, as a disease of elderly age, is also important due to its various possible consequences and significant financial burden, and the timely use of the cost-effectiveness screening strategies for timely intervention will have great importance.

Based on our knowledge, the designed economic model in present study is the first full economic evaluation model to compare sarcopenia screening strategies, which due to all limitations of information, tried to be designed and implemented systematically and step by step based on the best available evidences. Previous studies have focused more on the clinical features of the sarcopenia and in some cases the economic costs of the disease, such as the study of Janssen et al. (2004) and Goates et al. (2019) in the US [33, 52] and the study of Sousa et al. (2016) in Portugal [53].

As mentioned in this model, according to the available evidence, reducing the quality of life, falling, fracture and increased risk of CVDs were considered as clinical outcomes of the disease and other outcomes were ignored due to information constraints. Also in this model, due to lack of access to classified evidence, the division of sarcopenia into pre sarcopenia and severe sarcopenia subgroup was avoided. Another limitation we encountered in designing and implementing the model was the efficacy of treatment and sarcopenia interventions. Since the specific drug has not yet been approved for the treatment and reduction of sarcopenia complications, the efficacy of treatment in this study was based solely on endurance exercises and dietary supplements.

By expanding the evidence and gaining more access to the required parameters and removing study constraints, more complete models can be designed and implemented in the future to identify the most effective strategies.

\section{Conclusions}

The results of the study, as the first economic evaluation of sarcopenia screening, showed that the EWGSOP strategy is more cost-effective than other strategies. Also, Sensitivity analysis showed that the base case results were highly robust.

\section{Abbreviations}

CEA: Cost Effectiveness Analysis; SarSA-Mod: Sarcopenia Scoring Assessment Models; EWGSOP: European Working Group on Sarcopenia in Older People; MSRA: Mini Sarcopenia Risk Assessment; AWGS: Asian Working Group for

Sarcopenia; QALYs: Quality-Adjusted Life Years; CVDs: Cardiovascular Diseases; BIA: Bio-impedance Analysis; DXA: Dual-energy X-ray absorptiometry; ROC: Receiver Operating Characteristic; ICER: Incremental Cost-Effectiveness Ratio; DSA: Deterministic Sensitivity Analysis; PSA: Probabilistic Sensitivity Analysis; CEAC: Cost Effectiveness Acceptability Curve

\section{Supplementary Information}

The online version contains supplementary material available at https://doi. org/10.1186/s12889-021-10511-7.

Additional file 1: Figure S1. Prisma Diagram.

Additional file 2: Table S1. Comparison between SarSA-Mod, SARC-F and EWGSOP.

\section{Acknowledgements}

Not applicable.

\section{Authors' contributions}

$A D$ and GSh were in charge of collecting the data. MR and GSh took the lead in writing the article. GSh and $\mathrm{RH}$ were in charge of clinical and specialized research sections. AD, MR, RD and FH did the modelling and analyses. MR and MM performed the Systematic review. AD and MR helped with interpreting the outcomes and edition the article. All authors read, edited and approved the final article.

\section{Funding}

Not applicable.

Availability of data and materials

All data generated or analysed during this study are included in this published article [and its supplementary information files]. The datasets used and/or analysed during the current study are available from the corresponding author on reasonable request.

\section{Declarations}

Consent to publication

Not applicable.

Ethics approval and consent to participate

This research was approved by the ethical committee of Tehran University of Medical Sciences (TUMS) by the code of IR.TUMS.EMRI.REC.1397.055.

Competing interests

There are no conflicts of interest that are directly relevant to the content of this article.

\footnotetext{
Author details

${ }^{1}$ Chronic Diseases Research Center, Endocrinology and Metabolism Population Sciences Institute, Tehran University of Medical Sciences (TUMS), NO 10, Jalale-Al-Ahmad Ave, Chamran Highway, Tehran 1411713137, Iran. ${ }^{2}$ Department of Health Management and Economics, School of Public Health, Tehran University of Medical Sciences, Poursina AVE, Tehran 1417613151, Iran. ${ }^{3}$ Aberdeen Centre for Health Data Sciences, University of Aberdeen, Foresthill, Aberdeen AB252ZD, UK. ${ }^{4}$ National Center for Health Insurance Research, Iran Health Insurance Organization, Tehran, Iran. ${ }^{5}$ Department of Mathematics, Faculty of Sciences, Shahid Rajaee Teacher Training University, Lavizan, Tehran 1417613363, Iran.
} 


\section{Received: 26 September 2020 Accepted: 26 February 2021}

\section{Published online: 17 May 2021}

\section{References}

1. As' habi A, Najafi I, Tabibi H, Hedayati M. Prevalence of sarcopenia and dynapenia and their determinants in Iranian peritoneal dialysis patients. Iranian J Kidney Dis. 2018;12(1):53.

2. Heshmat R, Shafiee G, Shadman Z, Hashemi R, Khatami F, Ahadi Z, et al. A comprehensive manual on sarcopenia. 1st ed. Noavaran Sina: Tehran; 2017.

3. Karakelides H, Nair KS. Sarcopenia of aging and its metabolic impact. Curr Top Dev Biol. 2005;68:123-48

4. Narici MV, Reeves ND, Morse Cl, Maganaris CN. Muscular adaptations to resistance exercise in the elderly. J Musculoskelet Nueronal Interact. 2004; 4(2):161-4.

5. Lovell DI, Cuneo R, Gass GC. The effect of strength training and short-term detraining on maximum force and the rate of force development of older men. Eur J Appl Physiol. 2010;109(3):429-35.

6. Yarasheski KE, Pak-Loduca J, Hasten DL, Obert KA, Brown MB, Sinacore DR. Resistance exercise training increases mixed muscle protein synthesis rate in frail women and men $\geq 76$ yr old. Am J Physiol Endocrinol Metabol. 1999; 277(1):E118-E25

7. Delmonico MJ, Harris TB, Lee JS, Visser M, Nevitt M, Kritchevsky SB, et al. Alternative definitions of sarcopenia, lower extremity performance, and functional impairment with aging in older men and women. J Am Geriatr Soc. 2007;55(5):769-74.

8. Goodpaster BH, Park SW, Harris TB, Kritchevsky SB, Nevitt M, Schwartz AV, et al. The loss of skeletal muscle strength, mass, and quality in older adults: the health, aging and body composition study. J Gerontol Ser A Biol Med Sci. 2006;61(10):1059-64.

9. Binkley N, Krueger D, Buehring B. What's in a name revisited: should osteoporosis and sarcopenia be considered components of "dysmobility syndrome?". Osteoporos Int. 2013;24(12):2955-9.

10. Chien M-Y, Kuo H-K, Wu Y-T. Sarcopenia, cardiopulmonary fitness, and physical disability in community-dwelling elderly people. Phys Ther. 2010; 90(9):1277-87.

11. Chin SO, Rhee SY, Chon S, Hwang Y-C, Jeong I-K, Oh S, et al. Sarcopenia is independently associated with cardiovascular disease in older Korean adults: the Korea National Health and Nutrition Examination Survey (KNHANES) from 2009. PLoS One. 2013;8(3):e60119.

12. Fahimfar N, Tajrishi FZ, Gharibzadeh S, Shafiee G, Tanha K, Heshmat R, et al. Prevalence of osteosarcopenia and its association with cardiovascular risk factors in Iranian older people: Bushehr elderly health (BEH) program. Calcif Tissue Int. 2020;106(4):364-70.

13. Janssen I, Heymsfield SB, Ross R. Low relative skeletal muscle mass (sarcopenia) in older persons is associated with functional impairment and physical disability. J Am Geriatr Soc. 2002;50(5):889-96.

14. Shafiee G, Keshtkar A, Soltani A, Ahadi Z, Larijani B, Heshmat R. Prevalence of sarcopenia in the world: a systematic review and metaanalysis of general population studies. J Diabet Metabol Disord. 2017; 16(1):21.

15. Van Kan GA. Epidemiology and consequences of sarcopenia. JNHA. 2009; 13(8):708-12.

16. Volpato S, Bianchi L, Cherubini A, Landi F, Maggio M, Savino E, et al. Prevalence and clinical correlates of sarcopenia in community-dwelling older people: application of the EWGSOP definition and diagnostic algorithm. J Gerontol A Biol Sci Med Sci. 2014;69(4):438-46.

17. Cherin P, Voronska E, Fraoucene N, de Jaeger C. Prevalence of sarcopenia among healthy ambulatory subjects: the sarcopenia begins from 45 years. Aging Clin Exp Res. 2014;26(2):137-46.

18. Cerri AP, Bellelli G, Mazzone A, Pittella F, Landi F, Zambon A, et al. Sarcopenia and malnutrition in acutely ill hospitalized elderly: prevalence and outcomes. Clin Nutr. 2015;34(4):745-51.

19. Gariballa S, Alessa A. Sarcopenia: prevalence and prognostic significance in hospitalized patients. Clin Nutr. 2013 Oct;32(5):772-6.

20. Rossi AP, Fantin F, Micciolo R, Bertocchi M, Bertassello P, Zanandrea V, et al. Identifying sarcopenia in acute care setting patients. J Am Med Direct Assoc. 2014;15(4):303. e7-e12

21. Sousa AS, Guerra RS, Fonseca I, Pichel F, Amaral TF. Sarcopenia among hospitalized patients-a cross-sectional study. Clin Nutr. 2015; 34(6):1239-44.
22. Hashemi R, Heshmat R, Motlagh AD, Payab M, Esmaillzadeh A, Baigy F, et al. Sarcopenia and its determinants among Iranian elderly (SARIR): study protocol. J Diabet Metabol Disord. 2012;11(1):23.

23. Hashemi R, Shafiee G, Motlagh AD, Pasalar P, Esmailzadeh A, Siassi F, et al. Sarcopenia and its associated factors in Iranian older individuals: results of SARIR study. Arch Gerontol Geriatr. 2016;66:18-22.

24. Shafiee G, Heshmat R, Ostovar A, Nabipour I, Larijani B. Sarcopenia disease in Iran: an overview. J Diabet Metabol Disord. 2019;18:665-74.

25. Hashemi R, Motlagh AD, Heshmat R, Esmaillzadeh A, Payab M, Yousefinia M, et al. Diet and its relationship to sarcopenia in community dwelling Iranian elderly: a cross sectional study. Nutrition. 2015;31(1):97-104.

26. Ishii S, Tanaka T, Shibasaki K, Ouchi Y, Kikutani T, Higashiguchi T, et al. Development of a simple screening test for sarcopenia in older adults. Geriatr Gerontol Int. 2014;14:93-101.

27. Larijani B, Shafiee G, Shadman Z, Ostovar A, Heshmat R, Taheri E, et al. Association between nutritional status and sarcopenia in a community dwelling older population: The Bushehr elderly health (BEH) program. J Bone Min Res. 2018;33(supp 1):439.

28. Cruz-Jentoft A, Baeyens J, Bauer J, Cruz-Jentoft A, Boirie Y, Cederholm T, European Working Group on Sarcopenia in Older People, et al. Sarcopenia: European consensus on definition and diagnosis: report of the European Working Group on Sarcopenia in Older People. Age Ageing. 2010;39:412-23.

29. Malmstrom TK, Morley JE. SARC-F: a simple questionnaire to rapidly diagnose sarcopenia. J Am Med Dir Assoc. 2013;14(8):531-2.

30. Yu S, Appleton S, Chapman I, Adams R, Wittert G, Visvanathan T, et al. An anthropometric prediction equation for appendicular skeletal muscle mass in combination with a measure of muscle function to screen for sarcopenia in primary and aged care. J Am Med Dir Assoc. 2015;16(1): 25-30.

31. Rossi AP, Micciolo R, Rubele S, Fantin F, Caliari C, Zoico E, et al. Assessing the risk of sarcopenia in the elderly: the mini sarcopenia risk assessment (MSRA) questionnaire. J Nutr Health Aging. 2017;21(6):743-9.

32. Shafiee GHR, Ostovar A, Keshtkar A, Nabipour I, Larijani B. Development and validation of sarcopenia screening model for older people: the bushehr elderly health program. In: World Congress on Osteoporosis, Osteoarthritis and Musculoskeletal Diseases (WCO-IOF-ESCEO 2018). Poland: Osteoporosis International; 2018. p. s329-30. https://doi.org/10.1 007/s00198-018-4465-1.

33. Janssen I, Shepard DS, Katzmarzyk PT, Roubenoff R. The healthcare costs of sarcopenia in the United States. J Am Geriatr Soc. 2004;52(1):80-5.

34. Heshmat R, Hashemi R, Motlagh A, Payab M, Esmailzadeh A, Pasalar P, et al. Presarcopenia, sarcopenia and severesarcopenia among Iranian elderlies: The first epidemiological report. Osteoporosis International. England: Springer London LTD 236 grays INN RD; 2014

35. Cruz-Jentoft A, Baeyens J, Bauer J, Cruz-Jentoft A, Boirie Y, Cederholm T, et al. European working group on sarcopenia in older people. Sarcopenia: European consensus on definition and diagnosis: report of the European working group on sarcopenia in older people. Age Ageing. 2010;39:412-23.

36. Shafiee G, Heshmat R, Ostovar A, Khatami F, Fahimfar N, Arzaghi SM, et al. Comparison of EWGSOP-1and EWGSOP-2 diagnostic criteria on prevalence of and risk factors for sarcopenia among Iranian older people: the Bushehr elderly health (BEH) program. J Diabet Metabol Disord. 2020;19:727-34. https://doi.org/10.1007/s40200-020-00553-w.

37. Saka B, Ozkaya H, Karisik E, Akin S, Akpinar T, Tufan F, et al. Malnutrition and sarcopenia are associated with increased mortality rate in nursing home residents: a prospective study. Eur Geriatr Med. 2016;7(3):232-8.

38. Yalcin A, Aras S, Atmis V, Cengiz OK, Cinar E, Atli T, et al. Sarcopenia and mortality in older people living in a nursing home in Turkey. Geriatr Gerontol Int. 2017;17(7):1118-24.

39. Beaudart C, Zaaria M, Pasleau F, Reginster J-Y, Bruyère O. Health outcomes of sarcopenia: a systematic review and meta-analysis. PloS one. 2017;12(1): e0169548.

40. Landi F, Liperoti R, Russo A, Giovannini S, Tosato M, Capoluongo E, et al. Sarcopenia as a risk factor for falls in elderly individuals: results from the ilSIRENTE study. Clin Nutr. 2012;31(5):652-8.

41. Shafiee G, Heshmat R, Ostovar A, Sharifi F, Razi F, Nabipour I, et al. Association of sarcopenia and its parameters with type 2 diabetes: the bushehr elderly health program. Osteoporosis International. England: Springer London LTD 236 grays INN RD; 2014 
42. Goudarzi R, Sari AA, Zeraati H, Rashidian A, Mohammad K, Amini S. Valuation of quality weights for EuroQol 5-dimensional health states with the time trade-off method in the capital of Iran. Value Health Region Issues. 2019;18:170-5.

43. Goudarzi R, Zeraati H, Sari AA, Rashidian A, Mohammad K. Population-based preference weights for the EQ-5D health states using the visual analogue scale (VAS) in Iran. Iran Red Crescent Med J. 2016;18(2):e21584.

44. Der Sheng Sun HL, Yim HW, Won HS, Ko YH. The impact of sarcopenia on health-related quality of life in elderly people: Korean National Health and nutrition examination survey. Korean J Intern Med. 2019;34(4):877.

45. Peasgood T, Herrmann K, Kanis J, Brazier J. An updated systematic review of health state utility values for osteoporosis related conditions. Osteoporos Int. 2009;20(6):853.

46. Azami-Aghdash S, Gharaee H, Aghaei MH, Derakhshani N. Cardiovascular diseases patient's quality of life in Tabriz-Iran: 2018. J Commun Health Res. 2019;8(4):245-52.

47. Hernlund E, Svedbom A, Ivergård M, Compston J, Cooper C, Stenmark J, et al. Osteoporosis in the European Union: medical management, epidemiology and economic burden. Arch Osteoporos. 2013;8(1-2):136.

48. Damanti S, Azzolino D, Roncaglione C, Arosio B, Rossi P, Cesari M. Efficacy of nutritional interventions as stand-alone or synergistic treatments with exercise for the Management of Sarcopenia. Nutrients. 2019;11(9):1991.

49. Steffl M, Bohannon RW, Sontakova L, Tufano JJ, Shiells K, Holmerova I. Relationship between sarcopenia and physical activity in older people: a systematic review and meta-analysis. Clin Interv Aging. 2017;12:835.

50. Yoshimura Y, Wakabayashi H, Yamada M, Kim H, Harada A, Arai H. Interventions for treating sarcopenia: a systematic review and meta-analysis of randomized controlled studies. J Am Med Direct Assoc. 2017;18(6):553. e1-e16.

51. Drummond MF, Sculpher MJ, Claxton K, Stoddart GL, Torrance GW. Methods for the economic evaluation of health care programmes. 4th ed. Oxford: Oxford University Press; 2015.

52. Goates S, Du K, Arensberg M, Gaillard T, Guralnik J, Pereira SL. Economic impact of hospitalizations in US adults with sarcopenia. J Frailty Aging. 2019;8(2):93-9. https://doi.org/10.14283/jfa.2019.10.

53. Sousa AS, Guerra RS, Fonseca I, et al. Financial impact of sarcopenia on hospitalization costs. Eur J Clin Nutr. 2016;70:1046-51. https://doi.org/10.103 8/ejcn.2016.73.

\section{Publisher's Note}

Springer Nature remains neutral with regard to jurisdictional claims in published maps and institutional affiliations.

\section{Ready to submit your research? Choose BMC and benefit from:}

- fast, convenient online submission

- thorough peer review by experienced researchers in your field

- rapid publication on acceptance

- support for research data, including large and complex data types

- gold Open Access which fosters wider collaboration and increased citations

- maximum visibility for your research: over $100 \mathrm{M}$ website views per year

At BMC, research is always in progress.

Learn more biomedcentral.com/submissions 